\title{
The protective effect of microRNA-21 in neurons after spinal cord injury
}

\author{
Tao Zhang ${ }^{1,2} \cdot$ Shuangfei $\mathrm{Ni}^{3} \cdot$ Zixiang $\mathrm{Luo}^{3} \cdot$ Ye Lang ${ }^{3} \cdot$ Jianzhong $\mathrm{Hu}^{2,3} \cdot \mathrm{Hongbin}^{\mathrm{Lu}} \mathrm{Lu}^{1,2}$
}

Received: 19 December 2017 / Revised: 25 June 2018 / Accepted: 25 June 2018 / Published online: 8 August 2018

(c) The Author(s) 2018. This article is published with open access

\begin{abstract}
Study design Experimental animal study.

Objectives To validate the anti-apoptosis effect of microRNA-21 in neurons after spinal cord injury (SCI) and explore the mechanism.

Setting Xiangya Hospital, Central South University, Changsha, Hunan, People's Republic of China.

Methods In situ hybridization was used to detect the expression of miR-21 in spinal cord neurons $(n=24)$. In a rat contusion SCI model $(n=48)$, we upregulated the miR-21 level around the injured area using miR-21 lentiviral vectors and evaluated the therapeutic effect with histology and behavioural scores. In neuronal cells, oxygen-glucose deprivation (OGD) was exerted to imitate SCI, and we explored the biomechanism using molecular biology and a dual-luciferase reporter assay. Results miR-21 was expressed in spinal cord neurons and was found to improve neuronal survival and promote functional recovery in rat SCI models. The in vitro results in PC-12 cells revealed that the augmentation of endogenous miR-21 was able to reduce neuronal cell death after OGD. In addition, overexpression of miR-21 was able to reduce cellular apoptosis via decreasing PDCD4 protein levels, and caspase-3 activity was also influenced. Transfection of miR-21 into 293T cells was able to decrease luciferase activity in a reporter assay system, including the $3^{\prime}$ untranslated region of PDCD4.

Conclusions miR-21 may have a protective role in neuronal apoptosis after SCI. PDCD4 may be a functional target gene involved in the miR-21-mediated anti-apoptotic effect through an miR-21/PDCD4/caspase-3 pathway.
\end{abstract}

\section{Introduction}

To treat spinal cord injury (SCI) more effectively, many basic and clinical investigators focus on the underlying pathological mechanisms of SCI, which involve primary mechanical injury and secondary injury. During secondary

These authors contributed equally: Jianzhong Hu, Hongbin Lu.

Hongbin Lu

hongbinlu@hotmail.com

1 Department of Sports Medicine, Research Center of Sports Medicine, Xiangya Hospital, Central South University, Changsha, Hunan, People's Republic of China

2 Key Laboratory of Organ Injury, Aging and Regenerative Medicine of Hunan Province, Changsha, Hunan, People's Republic of China

3 Department of Spine Surgery, Xiangya Hospital, Central South University, Changsha, Hunan, People's Republic of China injury, ischaemia/hypoxia and reperfusion are some of the key pathophysiological changes and lead to a series of apoptotic neuronal cell death. Neurons in the injured boundary zone may be rescued, and the survival of these neurons is crucial for functional rehabilitation [1, 2]. However, the potential molecular mechanisms that determine neuronal apoptosis are still not entirely clear.

miRNAs are endogenous non-coding RNAs (18-22 nucleotides) that can negatively regulate gene expression at the post-transcriptional level [3]. To date, several miRNAs have been detected in the mammalian brain and spinal cord, and they seem to play vital roles in some neurological functions [4, 5]. In particular, miR-21 is a strong antiapoptotic gene in solid tumours [6] that regulates the expression of certain genes, such as PDCD4 and PTEN. Furthermore, it was reported that miR-21 is upregulated in some types of central nervous system injuries, such as stroke and traumatic brain injury [7, 8]. Previously, we found using miRNA arrays that miR-21 was one of the most dysregulated miRNAs after SCI in a rat model, and knockdown of miR-21 by an antagomir was correlated with 
apoptosis and functional deficits [9]. Nevertheless, the relevant biological roles of miR-21 in spinal cord neurons remain poorly understood and require further study.

In the present study, we measured miR-21 expression in neurons from the rat contused spinal cord and PC-12 neuroblastoma cell lines, which underwent oxygen-glucose deprivation (OGD). To investigate the biologic effect of miR-21 in SCI neurons, we artificially overexpressed miR-21 via lentiviral vectors. Furthermore, we explored the potential mechanisms by examining PDCD4, which was associated with ischaemia/hypoxia reperfusion-induced apoptosis [10]. PDCD4 is thought to induce apoptosis by activating the caspase-3 pathway [11]. Our results indicate that miR-21 is upregulated in neurons after SCI, and it appears to be able to reduce neuronal sensitivity to apoptosis by targeting PDCD4.

\section{Methods}

\section{Lentiviral vector construction}

The lentiviral vectors were constructed by GeneChem (Shanghai, China) to artificially overexpress miR-21, and a green fluorescent protein (GFP) tag was encoded in the vector sequence. The sequence of mir-21(6648-1)-P1 was AGCTGTACAAGTAAGTGGCATTAAGCCCCGGCAAG, and the sequence of mir-21(6648-1)-P2 was GGGAGA GGGGCTTAGTGCAAGTCTCATGAGACATAAG. The pre-miR-21 hairpin structure and scramble control hairpin were inserted in the lentiviral expression vector GV254 (Ubi-EGFP-MCS-IRES-Puromycin), which was provided by GeneChem (Shanghai, China) using a Nhel site. Packaging of the GV254 miR-21 lentivirus particles was completed in a pseudoviral particle producer cell line (293 TN cells) by co-transfecting with the envelope plasmids Pmd2.G and pPAX using Lipofectamine 2000 (Invitrogen, Carlsbad, CA, USA). The supernatant was harvested 48 and $72 \mathrm{~h}$ after transfection. The viral particles were further concentrated, as described previously [12]. An empty vector was used as a negative control.

\section{Animal model of SCI}

Adult male Sprague-Dawley (SD) rats weighing 180-220 g were anaesthetized by $10 \%$ chloral hydrate $(3 \mathrm{mg} / \mathrm{kg})$. Moderate SCI models $(n=64)$ were induced by a modified Allen's weight drop apparatus at the thoracic vertebra level 10. After the rats were anaesthetized, an incision was made on the dorsomedial line, and the spine was exposed clearly centred at the T10 vertebra. A laminectomy was carefully performed to reveal the spinal dura mater, approximately 3 $\mathrm{mm}$ in length. Then, moderate contusion injury was induced by a modified Allen's weight drop apparatus ( $8 \mathrm{~g}$ weight at a vertical height of $40 \mathrm{~mm}, 8 \mathrm{~g} \times 40 \mathrm{~mm}$ ). Successful signs of a rat SCI model were as follows: bleeding and oedema in the T10 spinal dura mater, retracted flapping of both hind limbs, spasmodic sway of the tail, and flaccid paralysis after palinesthesia. Uninjured animals $(n=12)$ were subjected only to laminectomy. A total of eight uninjured and $16 \mathrm{SCI}$ rats were allocated for in situ hybridization (ISH) and laser capture microdissection (LCM). The other 48 SCI rats were randomly divided into two groups: the LV-miR21 group $(n=24)$ and the negative control (LV-NC) group $(n=24)$. Each animal in the two groups received $5 \mu \mathrm{l}$ of the respective implant (LV/miR-21-shRNA suspension for the LV-miR21 group and NC-LV suspension for LV-NC group) at the rostral and caudal areas of the cord around the lesion $(2.5 \mu \mathrm{l}$ at each point). Post-operative care of rats included analgesia $(0.25 \%$ Bupivacaine, subcutaneous, $0.1 \mathrm{ml})$ and daily manual bladder training until the reflexive control of bladder function was restored. At different time points, animals were euthanized with an overdose of $10 \%$ chloral hydrate $(10 \mathrm{mg} /$ $\mathrm{kg}$ ), and a 10-mm segment of spinal cord encompassing the injured site was harvested for subsequent testing.

\section{Basso, Beattie and Bresnahan (BBB) score}

Functional recovery was evaluated at 7 and 14 days postsurgery using the BBB open field locomotor test, as described previously [13]. The behaviours of the trunk, tail and hindlimbs of the rats were assessed in an open field. The observers were blind to the treatment.

\section{Tissue preparation}

Spinal cord tissues were harvested through three methods. For total protein extraction, the spinal cord segments were directly separated after euthanasia and then immediately preserved in liquid nitrogen. For cryosectioning, rats were terminally anaesthetized and transcardially perfused with $37^{\circ} \mathrm{C}$ heparinized saline, followed by $4 \%$ paraformaldehyde (PFA). The spinal cord tissue around the injured site was removed carefully, post-fixed in $4 \%$ PFA overnight at $4{ }^{\circ} \mathrm{C}$, and dehydrated in 15 and $30 \%$ sucrose solution until sinking. Next, the tissue was embedded in an optimal cutting temperature (OCT) and frozen at $-20^{\circ} \mathrm{C}$. The frozen tissue was transected and vertically sectioned in $10 \mu \mathrm{m}$ sections for ISH and LCM. For paraffin sectioning, the spinal cord tissue was embedded in paraffin after post-fixing overnight. Transverse sections $(10 \mu \mathrm{m})$ were taken for Nissl staining.

\section{ISH and immunofluorescence staining}

To verify whether miR-21 is expressed in spinal cord neurons, ISH was performed on transverse frozen sections 
using the 30\% LNA-70\% DNA3'-DIG-labelled mercury probes (Exiqon, Vedbaedk, Denmark), according to the manufacturer's protocol. Hybridized miR-21 was visualized with Alkaline Phosphatase (AP). After ISH, sections were blocked in PBS containing 0.5\% BSA and $0.05 \%$ Tween 20 for immunostaining. Incubation with a NeuN antibody (1:500; Abcam) was followed by incubation with a fluorescent secondary antibody. Finally, cells were stained with DAPI (1:7500, Sigma, USA) for $10 \mathrm{~min}$ to visualize nuclei.

\section{LCM}

LCM was used to separate neurons from the spinal cord to detect miR-21 in only spinal cord neurons by Real TimePolymerase Chain Reaction (RT-PCR). Transverse frozen sections $(10 \mu \mathrm{m})$ of spinal cord samples were mounted on LCM membrane slides and immunostained with an antibody against NeuN (1:250; Abcam), as previously reported [14]. Approximately 1000 NeuN-positive cells from each animal were captured on a thermoplastic film using the laser microdissection system (Leica LMD 6 500/7000, Wetzlar, Germany). Cells were transferred to Eppendorf tubes containing $300 \mu \mathrm{l}$ lysis buffer and stored at $-80^{\circ} \mathrm{C}$ before RNA isolation.

\section{Nissl staining and cell counts}

The area of the spinal cord 3-5 mm rostral and caudal to the injury (adjacent area) was transversely sectioned (10 $\mu \mathrm{m})$ with adjacent sections $200 \mu \mathrm{m}$ apart from each other. Sections were stained with Nissl Solution (Beyotime), and six sections (three rostral and three caudal) were counted from each rat. In each section, 10 squares (area $=0.01 \mathrm{~mm}^{2}$ ) were selected at $400 \times$ magnification to count the neuron number, including four squares in the anterior horn, four squares in the posterior horn and two squares around the myelocoele. During the counting procedure, only neurons whose nuclei were cut and visible were counted. After calculating the area of the grey matter using IPP (Image-Pro Plus 5.0.2; Media Cybernetics Inc.), we counted the neuron number in the grey matter. The grey matter and white matter were separated by the positive results, which were higher in the grey matter. The number of positively stained cells in the entire grey matter area was counted and averaged per section in a blind manner.

\section{Cell culture and transfection}

PC-12 cells were purchased from the Cell Storage Center of Wuhan University (Wuhan, China). Native PC-12 cells were maintained in the Roswell Park Memorial Institute (RPMI) 1640 medium (Gibco) that was supplemented with
$10 \%$ foetal bovine serum (FBS, Gibco), $5 \%$ horse serum (Gibco), $100 \mathrm{U} / \mathrm{ml}$ penicillin and $100 \mu \mathrm{g} / \mathrm{ml}$ streptomycin at $37{ }^{\circ} \mathrm{C}$ with $5 \% \mathrm{CO}_{2}$.

For miRNA transfection, cells were seeded in a six-well plate at $4 \times 10^{4}$ cells per well, and the lentiviral vectors with $8 \mu \mathrm{g} / \mathrm{ml}$ polybrene were added to the media $(\mathrm{MOI}=20)$, according to the protocol to infect PC-12 cells. After infection for $24 \mathrm{~h}$, the cells were selected using $3 \mu \mathrm{g} / \mathrm{ml}$ puromycin for 3 days. The living cells after puromycin selection were used for subsequent experiments. For PDCD4 transfection, the PDCD4 cDNA was transfected into PC-12 cells by Lipofectamine 2000 (Invitrogen, Carlsbad, CA, USA) following the manufacturer's instructions.

\section{OGD and reoxygenation}

For OGD, the stably transfected PC-12 cells were seeded into a six-well plate premixed with $50 \mathrm{ng} / \mathrm{ml}$ nerve growth factor (NGF $2.5 \mathrm{~S}$, Invitrogen) at a density of $4 \times 10^{4}$ cells/ well. After culturing at $37^{\circ} \mathrm{C}$ with $5 \% \mathrm{CO}_{2}$ for $72 \mathrm{~h}$, culture medium was replaced with glucose-free DMEM (Gibco), and cells were placed into an anaerobic chamber suffused with $94 \% \mathrm{~N}_{2} / 1 \% \quad \mathrm{O}_{2} / 5 \% \mathrm{CO}_{2}$ for $4 \mathrm{~h}$ at $37^{\circ} \mathrm{C}$. Subsequently, the cells were washed twice with RPMI 1640 and incubated for $24 \mathrm{~h}$ in their normal culture medium for the subsequent measurements.

\section{Apoptosis assay}

The level of apoptosis was measured by flow cytometry (FACS) using an annexin V-PE apoptosis detection kit (Beyotime), and caspase-3 activity was measured with a caspase-3 colorimetric assay kit (Keygen). For FACS, approximately 105 cells were harvested and incubated in $195 \mu \mathrm{l}$ binding buffer containing $5 \mu \mathrm{l}$ Annexin V-PE at $25^{\circ} \mathrm{C}$ for $10-20 \mathrm{~min}$, as recommended by the manufacturer. The detection of caspase- 3 activity was performed following the manufacturer's instructions, and the relative luminescence intensity was presented as the fold-change compared with untreated controls.

\section{Real-time semi-quantitative RT-PCR}

Total RNA from cultured PC-12 cells and spinal cord neurons separated by LCM were extracted using TRIzol (Invitrogen), according to the manufacturer's instructions. cDNA was prepared from total RNA using the PrimeScript RT reagent kit (TaKaRa), and qRT-PCR was performed using the SYBR Premix Ex Taq (TaKaRa). The relative miR-21 levels were normalized using U6 as an internal control. Although U6 was thought to be affected by 
Fig. $1 \mathrm{miR}-21$ was expressed in rat spinal cord neurons, and the expression was upregulated after SCI. a1-a3 The results of in situ hybridization showed that the expression of miR-21 increased after SCI in the injury boundary zone, especially on the seventh day after SCI (vertical sections). b1-b3 In situ hybridization and immunofluorescence revealed that miR-21 was co-localized with NeuN in some cells of the rat spinal cord on the seventh day after SCI (transections) (The region within the arc showed the rat spinal cord injury zone)

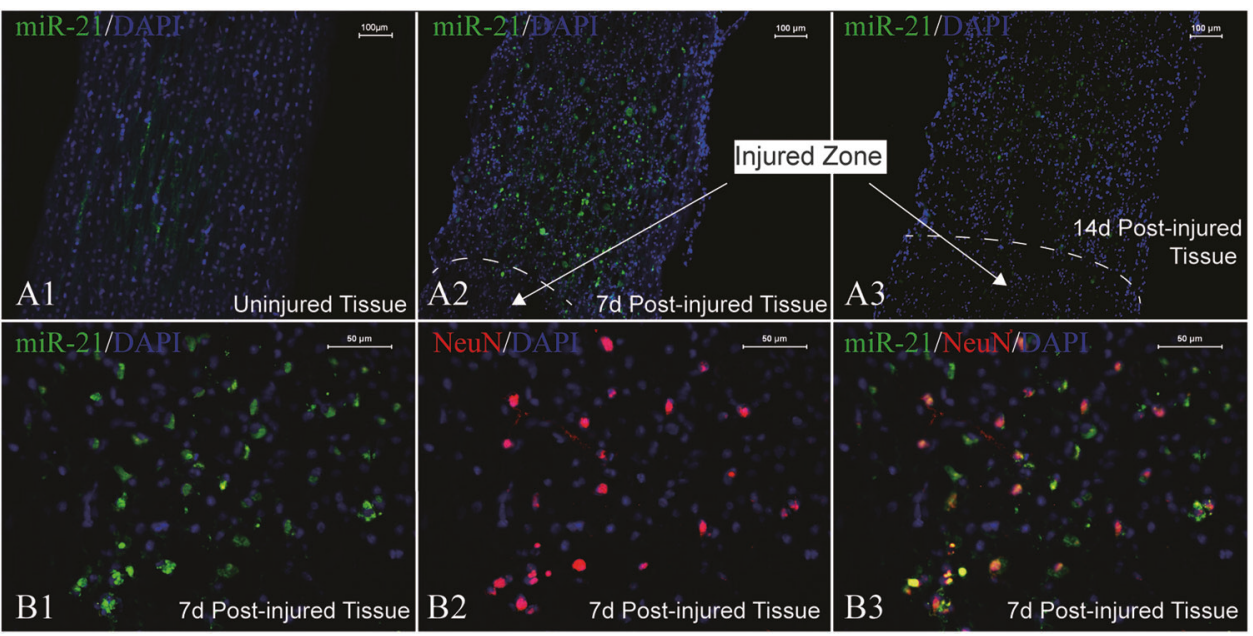

freeze-thaw cycles [15], it is a common reference and is still used in many studies. The analysis of gene expression was performed by the $2-\Delta \Delta \mathrm{CT}$ method.

\section{Western blotting}

The expression level of PDCD4, a target gene of miR-21, was determined by Western blot analysis. The freshly harvested spinal cord, including the injured spinal cord and the PC-12 cells harvested for Western blot analysis, were lysed, and the protein concentrations were determined using the BCA protein assay kit (Pierce). The protocol was performed as described previously [9]. Primary antibodies used in this study included an anti-rabbit-PDCD4 antibody (1:1000, Cell Signaling Technology) and an anti-GAPDH antibody (1:1000, Cell Signaling Technology).

\section{Dual-luciferase reporter assay}

To further confirm the targeted relationship between miR21 and PDCD4, a pMiR luciferase reporter assay with the PDCD4 3' untranslated region (3'UTR) (sequence: GGA GGGACCGAAGAGTAACCACCTATGTGTGGGGTGTT CTGATAAGCTACTTTCTAAGTG; mutant sequence: GG AGGGACCGAAGAGTAACCACCTATGTGTGGGGTGT GGATAATTTAGGCTTTCTAAGTG) was used for analysis (GeneChem Co. Ltd., Shanghai). Co-transfection of $293 \mathrm{~T}$ cells with PDCD4-3'UTR WT (3'UTR WT)/PDCD 4-3'UTR Mutant (3'UTR MU)/PDCD4-3'UTR negative control (3'UTR NC) and miR-21/miR-21 negative control (miR21-NC) in 24-well plates was completed using Lipofectamine 2000 (Invitrogen), according to the manufacturer's instructions. After $48 \mathrm{~h}$ of transfection, firefly and Renilla luciferase activities were analyzed with the luciferase reporter assay (Promega), according to the manufacturer's protocol.

\section{Statistics}

The data are all presented as the mean \pm SD and analyzed using SPSS 13.0 statistical software (SPSS Inc., Chicago, IL, USA). Differences between groups were analyzed by two-way ANOVA with Bonferroni's post hoc test or Student's $t$-test. A value of $p<0.05$ was considered significant.

\section{Results}

\section{miR-21 was upregulated in injured spinal cord neurons}

ISH was performed to investigate whether SCI affected miR-21 expression in neurons. In the uninjured spinal cord, miR-21 was detected at low levels, but abundant miR-21 signals were detected in cells bordering the lesion zone 7 days after SCI. At 14 day after SCI, miR-21 expression could still be detected, but it was lower than expression on the seventh day (Fig. 1. a1-a3). Moreover, to determine whether neurons express miR-21 in vivo following SCI, we co-stained NeuN protein, a marker of neurons. The immunostaining indicated that many cells with positive miR-21 signals were also NeuN-positive, but not all (Fig. 1. b1-b3). These data showed that miR-21 is upregulated in neurons around the lesion area.

However, miR-21 is not upregulated in neurons specifically, and ISH is not appropriate to quantify the gene expression. Therefore, neurons were isolated from the injured boundary zone of the injured spinal cord after SCI using LCM for RT-PCR analysis. The results revealed that there was a remarkable upregulation of miR-21 in injured neurons compared to those isolated from the uninjured spinal cord. miR-21 expression increased $3.68 \pm 0.18$-fold at 7 days post-injury $(n=4 ; p<0.05, t$-test $)$, and the 


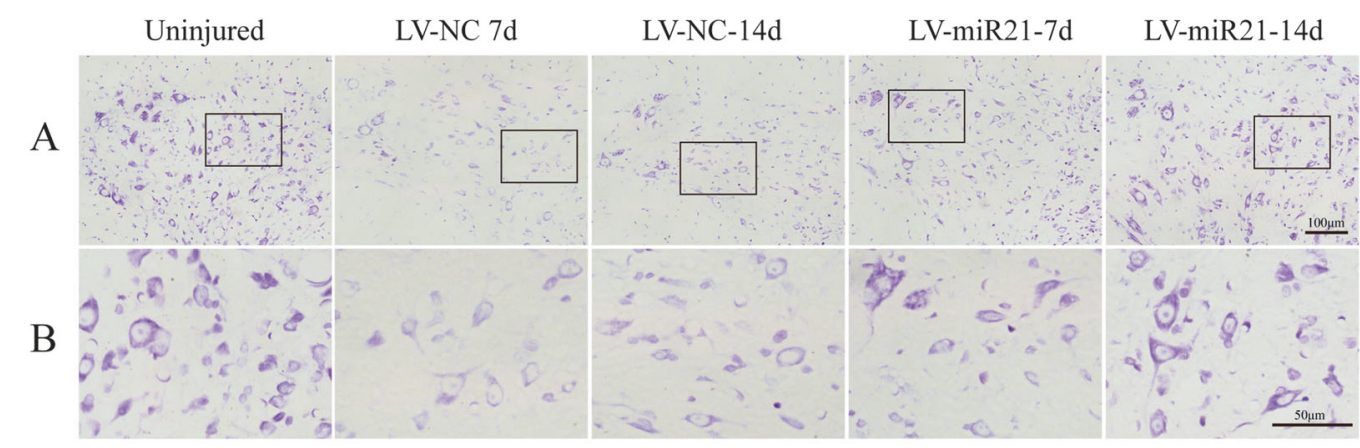

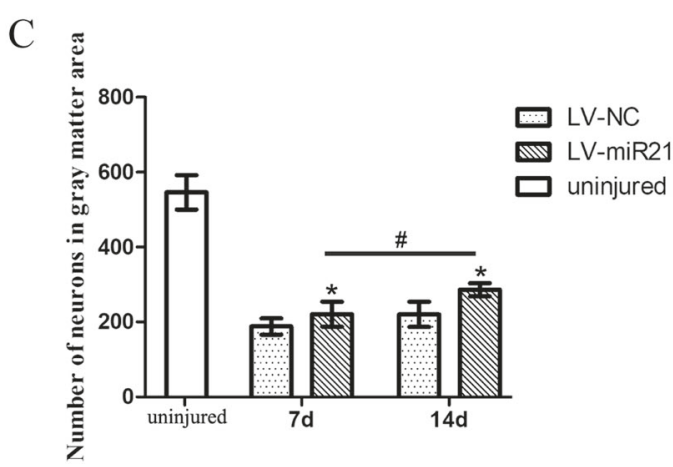

Fig. 2 LV-miR21 treatment was able to promote neuronal survival and functional recovery in the injured spinal cord. Neurons were visualized by Nissl staining in the boundary zone of the injured spinal cord in cross-sections, and the spinal cord function was evaluated by BBB scores (mean $\pm \mathrm{SD}$ ). a-c Representative images and quantitative analysis of surviving neurons by Nissl staining in different groups at

average increase in expression was approximately $3.22 \pm$ 0.16 times higher at 14 days after SCI $(n=4 ; p<0.05, t$ test), relative to the uninjured spinal cord. Therefore, these data suggested that miR-21 expression was significantly augmented in neurons after SCI.

\section{Positive role of miR-21 in neuronal survival in vivo}

In the present study, we investigated the effect of miR-21 on the neurons adjacent to the lesion site by Nissl staining at days 7 and 14 after SCI (Fig. 2a, b). The number of surviving neurons per section in the LV-miR21 group was significantly greater than that of the LV-NC group at the seventh $(n=4 ; 221 \pm 33$ vs. $188 \pm 21, p<0.05, t$-test $)$ and 14th day after injury $(n=4 ; 286 \pm 18$ vs. $220 \pm 34, p<0.05$, $t$-test) (Fig. 2c). In the LV-miR-21 group, there were a higher number of survival neurons at day 14 than at day 7 , while there was no significant difference in the LV-NC group. However, even in the miR-21 group on the 14th day following SCI, the number of surviving neurons was only approximately $52.4 \%$ of the number in the uninjured group. Moreover, we performed the BBB locomotor scale to evaluate whether miR-21 could improve functional recovery after SCI. After SCI, all rats exhibited complete paralysis in both hindlimbs (BBB score $=0)$, and hindlimb

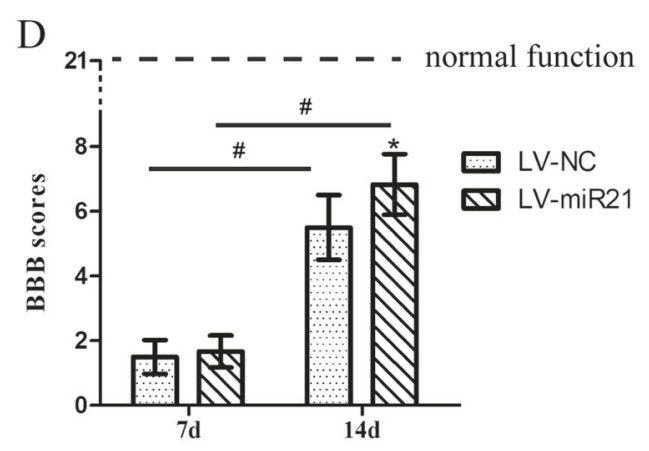

different time points; $\mathbf{b}$ shows a higher magnification of the boxed area in a. d The BBBs scores showed functional recovery in different groups at different time points $(n=4 ; * p<0.05$, the difference was significant between the LV-miR21 and LV-NC groups; ${ }^{*} p<0.05$, the difference was significant between the different time points)

locomotor function showed a gradual improvement with time in both groups (Fig. 2d). Compared to the scores of the LV-NC group, the BBB scores of the LV-miR21 animals were significantly higher at post-injury day $14(n=12$; $6.83 \pm 0.94$ vs. $5.50 \pm 1.00, p<0.05)$, while there was no difference at day $7(n=12 ; 1.67 \pm 0.49$ vs. $1.50 \pm 0.52$, $p<0.05$ ) (Two-way ANOVA: factor miR-21: $F(\mathrm{df}=1$ ) $=11.28, \quad p=0.0016 ;$ factor time: $F(\mathrm{df}=1)=421.15$, $p<0.001$; interaction: $F(\mathrm{df}=1)=6.82, p=0.0123)$.

\section{miR-21 decreased apoptosis of neurons in vitro}

To observe the dynamics of miR-21 in vitro, we used a model of ischaemia and reperfusion in which PC-12 cells were successively subjected to OGD and reoxygenation. We found that miR-21 expression increased significantly after OGD/reoxygenation for $24 \mathrm{~h}(2.48 \pm 0.15$-fold compared to expression in normal cells; $n=3 ; p<0.05, t$ test). We further evaluated the effect of miR-21 on the apoptosis of PC-12 cells using FACS analysis of annexin-V-stained cells. Overexpression of miR-21 decreased the percentage of annexin V-positive cells compared with the control group percentage (Table 1). Therefore, miR-21 was able to inhibit apoptosis in cultured PC-12 cells. 
Table 1 Cell apoptosis in response to the transfection of LV-miR21 and LV-NC determined by FACS analysis of annexin-V-stained cells and caspase- 3 activity (mean \pm standard deviation)

\begin{tabular}{lll}
\hline & LV-NC & LV-miR-21 \\
\hline Annexin-positive cells (\%) & $35.27 \pm 1.20$ & $22.37 \pm 1.79^{\mathrm{a}}$ \\
Relative caspase-3 activity & $1.00 \pm 0.16$ & $0.66 \pm 0.06^{\mathrm{a}}$ \\
\hline
\end{tabular}

${ }^{a} p<0.05$, difference was significant between the LV-miR21 and LVNC groups, $n=3$

Caspase- 3 is known to play a vital role in apoptosis. To determine whether the anti-apoptotic effect of miR-21 occurred via the pathway of caspase-3, we measured the caspase-3 activity. The results showed that caspase-3 enzymatic activity was reduced in LV-miR21 cells compared with activity in LV-NC cells (Table 1). Therefore, caspase-3 might be involved in the anti-apoptotic process of miR-21 after OGD/reoxygenation.

\section{PDCD4 expression was attenuated by miR-21 as a potential target gene}

Previous reports showed that miR-21 targeted PDCD4 and decreased its expression in some cancer cells [11]. However, the role of PDCD4 in neuronal apoptosis is currently unclear. In this study, PDCD4 was overexpressed by PDCD4 cDNA transfection in PC-12 cells $(n=3 ; 1.00 \pm 0.03$ vs. $2.24 \pm 0.06$, $p<0.05, t$-test, Fig. 3a), and the apoptosis rate of these cells increased after OGD $(n=3 ; 28.95 \pm 2.50$ vs. $39.43 \pm 1.76$, $p<0.05$, $t$-test, Fig. $3 \mathrm{~b}$ ). In PC-12 cells, upregulated miR-21 could decrease the expression of PDCD4, although PDCD4 levels increased after OGD in both the LV-NC $(n=3 ; 1.32 \pm$ 0.05 vs. $1.00 \pm 0.06, p<0.05)$ and LV-miR21 (0.69 \pm 0.10 vs. $0.56 \pm 0.07, p<0.05$ ) cells (Fig. $3 \mathrm{c}$ ) (Two-way ANOVA: factor miR-21: $F(\mathrm{df}=1)=223.95, p<0.001$; factor time: $F$ $(\mathrm{df}=1)=39.73, \quad p<0.001$; interaction: $F(\mathrm{df}=1)=6.66$, $p=0.024)$. In rat models, the expression of PDCD4 in the LV-miR21 group was also lower than in the LV-NC group at both the seventh day and 14th day after SCI $(n=4 ; p<0.05$, Fig. 3d) (Two-way ANOVA: factor miR-21: $F(\mathrm{df}=1)=$ 311.03, $p<0.001$; factor time: $F(\mathrm{df}=1)=0.56, p=0.467$; interaction: $F(\mathrm{df}=1)=0.96, p=0.3476)$. These data show that PDCD4 is a target gene of miR-21 involved in the mediation of neuronal apoptosis.

When we co-transfected $3^{\prime}$-UTR-WT with miR-21 in $293 \mathrm{~T}$ cells, the luciferase activity was only $72.8 \pm 1.4 \%$ compared to activity in cells co-transfected with miR21-NC ( $n=3 ; p<0.05, t$-test), while there was no difference in activity in the group transfected with $3^{\prime}$-UTR-MU $(n=3 ; p$ $>0.05, t$-test, Fig. 3e). These data therefore indicate that miR-21 could bind to the PDCD4 transcript via complementarity to its sequence.

\section{Discussion}

The present study confirmed that miR-21 was able to protect neurons after SCI in vitro and in vivo. miR-21 was expressed in neurons of the spinal cord and was significantly upregulated after injury. Overexpression of miR21 could decrease the apoptosis of neurons by inhibiting the PDCD4/caspase-3 pathway and improve the recovery of locomotor function.

Recently, various studies have reported that many miRNAs are involved in the prognosis of SCI through various pathways, including apoptosis, regeneration, inflammation and demyelination [16]. It is well known that apoptosis is considered to be a crucial mechanism of cell death in many neurological diseases and SCI [17]. The apoptotic process is associated with numerous genes, and therapeutic strategies might be able to prevent programmed cell death [18]. In particular, miR-21 has been shown to be an anti-apoptotic factor in many cancer cells [19], and its expression is significantly upregulated in some CNS diseases [7, 20]. Our previous study revealed that miR-21 was upregulated 3 days following rat SCI using miRNA microarray [9], and in a study by Oneil [21], miR-21 expression increased significantly at 5 weeks after SCI. However, these studies were both completed in animals and did not reveal whether miR21 was upregulated in neurons after SCI. Our present study demonstrated that miR-21 was expressed in neurons and was upregulated after rat SCI, but the expression was not specific.

Moreover, it has been shown that miR-21 has protective effects in the injury of different organs. In the injured region after myocardial infarction, miR-21 expression increased and played a protective role [22]. In addition, miR-21 was able to attenuate the apoptosis of cardiac cells induced by $\mathrm{H}_{2} \mathrm{O}_{2}$ [23], and it was involved in ischaemic preconditioning-mediated cardiac protection [24]. Moreover, miR-21 expression was also upregulated after traumatic brain injury [20, 25]. After stroke, the overexpression of miR-21 protected neurons from ischaemia by targeting Fasl [26]. In a rat SCI model, when miR-21 was downregulated by antagomir-21, apoptosis increased, and the expression of Fasl and PTEN increased [9]. In the present study, we found that overexpression of miR-21 decreased neuronal apoptosis and downregulated the expression of PDCD4.

PDCD4 is a pro-apoptotic gene and is able to regulate various aspects of many human diseases as a direct miR-21 target. In cancer, PDCD4 universally acts as a potential target for anticancer therapies [27]. In diabetes mellitus, PDCD4 is able to induce the apoptosis of pancreatic $\beta$-cells, and this process is controlled by miR-21 [28]. In heart ischaemia/reperfusion injury, PDCD4 is involved in cardiovascular biology by regulating the 
A

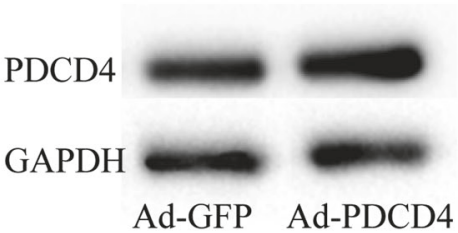

B

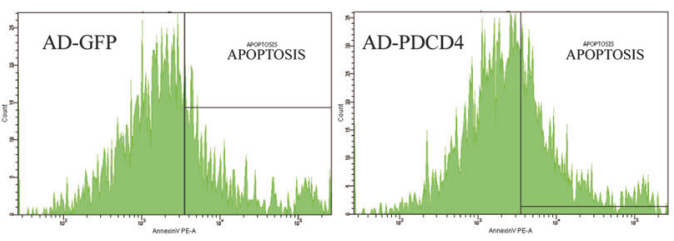

D
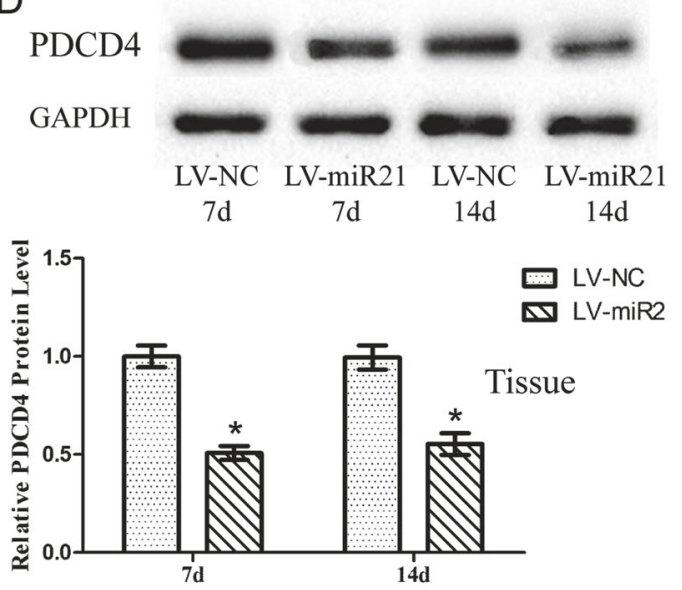

C
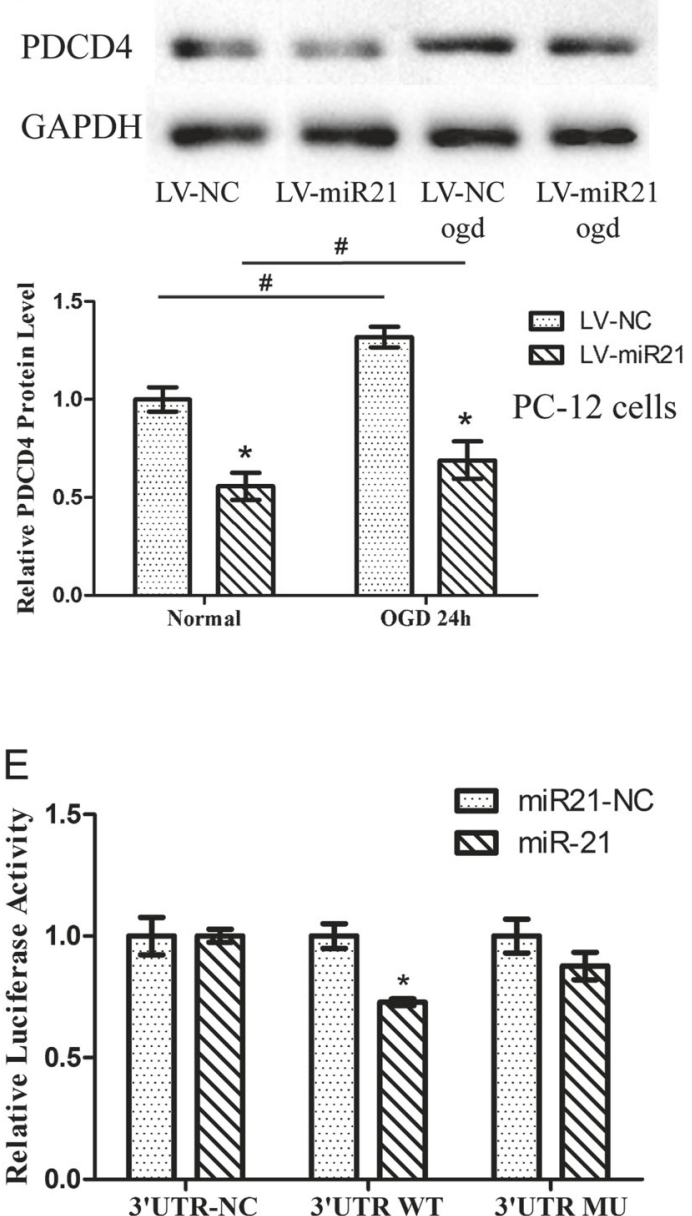

Fig. 3 PDCD4 is a direct target gene of miR-21 and is involved in the miR-21-mediated anti-apoptotic effect in PC-12 cells (mean \pm SD). a PDCD4 was overexpressed by adding PDCD4 cDNA to PC-12 cells. b Overexpression of PDCD4 resulted in a higher rate of apoptosis induced by OGD/reperfusion detected via FACS analysis. c-d Modulation of PDCD4 expression by LV-miR21 in PC-12 cells $(n=3)$ and in rats $(n=4)$; the overexpression of miR-21 was able to decrease the expression of PDCD4 $(* p<0.05$, the difference was significant between the LV-miR2 1 and LV-NC groups; $\# p<0.05$, the difference was significant between the different times in one group). e Validation of the binding sites of miR-21 in PDCD4 by a luciferase reporter assay. A fragment of the $3^{\prime}$-UTR of PDCD4 mRNA with the putative miR-21 binding sequence cloned into a firefly luciferase reporter and transfected into 293T cells together with miR-21 and miR21-NC. Firefly luciferase activity was normalized to Renilla luciferase expression $(n=3, * p<0.05$ compared with the miR21-NC group)

apoptosis of cardiac cells and vascular smooth muscle cells [24]. Caspases are the final effectors in the apoptotic pathway, and caspase-3 is a major mediator of apoptosis following injury [29]. PDCD4 is thought to at least partly drive apoptosis via the activation of the caspase-3 pathway [30]. Liu [31] showed that exercise leads to elevated levels of miR-21, which are correlated with changes in PDCD4 expression after SCI. Our experimental data showed that the miR-21/PDCD4/caspase-3 pathway participates in neuronal apoptosis in vitro and following $\mathrm{SCI}$ in vivo.

However, there were still some limitations in this study. The ischaemia/reperfusion injury model was established in PC-12 cells, not in primary cultured neurons. When treated with NGF, the PC-12 cells stop dividing and terminally differentiate into neuron-like cells. This makes PC-12 cells useful as a model to study the function and pathophysiology of neurons [32], and such a model has been used in SCI research [33]. We therefore thought its use was appropriate for this study. In addition, the mechanism of SCI is complex, and miR-21 was not the unique protective element during secondary SCI. Similarly, PDCD4 is not unique as a target gene of miR-21; there are other genes as well.

In summary, in rat SCI models and in the ischaemia/ reperfusion injury of PC-12 cells, miR-21 had a protective effect by reducing neuronal apoptosis via the miR-21/ PDCD4/caspase-3 pathway. Identifying the roles of miR-21 in neuronal protection might make it a novel therapeutic target for secondary SCI. 
Funding This work was supported by the National Natural Science Foundation of China (No. 81371956, No. 81171698 and No. 81672174).

Author contribution TZ was responsible for drafting and revising the article and conducting the research. SN contributed to conducting the research. ZL and YL contributed to data extraction and analysis. JH and HL were responsible for conceiving and designing the research and playing an important role in interpreting the results.

\section{Compliance with ethical standards}

Conflict of interest The authors declare that they have no conflict of interest.

Statement of ethics We certify that all applicable institutional and governmental regulations concerning the ethical use of animals were followed during the course of this research.

Open Access This article is licensed under a Creative Commons Attribution-NonCommercial-NoDerivatives 4.0 International License, which permits any non-commercial use, sharing, distribution and reproduction in any medium or format, as long as you give appropriate credit to the original author(s) and the source, and provide a link to the Creative Commons license. You do not have permission under this license to share adapted material derived from this article or parts of it. The images or other third party material in this article are included in the article's Creative Commons license, unless indicated otherwise in a credit line to the material. If material is not included in the article's Creative Commons license and your intended use is not permitted by statutory regulation or exceeds the permitted use, you will need to obtain permission directly from the copyright holder. To view a copy of this license, http://creativecommons.org/licenses/by-nc-nd/4.0/.

\section{References}

1. Nardone R, Pikija S, Mutzenbach JS, Seidl M, Leis S, Trinka E, et al. Current and emerging treatment options for spinal cord ischemia. Drug Discov Today. 2016;21:1632-41.

2. Shen Z, Zhou Z, Gao S, Guo Y, Gao K, Wang H, et al. Melatonin inhibits neural cell apoptosis and promotes locomotor recovery via activation of the Wnt/beta-catenin signaling pathway after spinal cord injury. Neurochem Res. 2017;42:2336-43.

3. Kosik KS. The neuronal microRNA system. Nat Rev Neurosci. 2006;7:911-20.

4. Bhalala OG, Srikanth M, Kessler JA. The emerging roles of microRNAs in CNS injuries. Nat Rev Neurol. 2013;9:328-39.

5. Hao N, Lu G, Liu X, Yu B, Wu H, Ji D, et al. Potential role of microRNA: identification and functional analysis of microRNA in corticospinal tract after unilateral lesions of the medullary pyramid. Neurosci Lett. 2014;564:37-42.

6. Jiang LP, He CY, Zhu ZT. Role of microRNA-21 in radiosensitivity in non-small cell lung cancer cells by targeting PDCD4 gene. Oncotarget. 2017;8:23675-89.

7. Zhang L, Dong LY, Li YJ, Hong Z, Wei WS. miR-21 represses FasL in microglia and protects against microgliamediated neuronal cell death following hypoxia/ischemia. Glia. 2012;60:1888-95.

8. Ge X, Han Z, Chen F, Wang H, Zhang B, Jiang R, et al. MiR-21 alleviates secondary blood-brain barrier damage after traumatic brain injury in rats. Brain Res. 2015;1603:150-7.
9. Hu JZ, Huang JH, Zeng L, Wang G, Cao M, Lu HB. Antiapoptotic effect of microRNA-21 after contusion spinal cord injury in rats. J Neurotrauma. 2013;30:1349-60.

10. Zhu J, Yao K, Wang Q, Guo J, Shi H, Ma L, et al. Ischemic postconditioning-regulated miR-499 protects the rat heart against ischemia/reperfusion injury by inhibiting apoptosis through PDCD4. Cell Physiol Biochem. 2016;39:2364-80.

11. White K, Dempsie Y, Caruso P, Wallace E, McDonald RA, Stevens $\mathrm{H}$, et al. Endothelial apoptosis in pulmonary hypertension is controlled by a microRNA/programmed cell death 4/caspase-3 axis. Hypertension. 2014;64:185-94.

12. Kutner RH, Zhang XY, Reiser J. Production, concentration and titration of pseudotyped HIV-1-based lentiviral vectors. Nat Protoc. 2009;4:495-505.

13. Basso DM, Beattie MS, Bresnahan JC. A sensitive and reliable locomotor rating scale for open field testing in rats. J Neurotrauma. 1995;12:1-21.

14. Liu XS, Chopp M, Zhang RL, Hozeska-Solgot A, Gregg SC, Buller B, et al. Angiopoietin 2 mediates the differentiation and migration of neural progenitor cells in the subventricular zone after stroke. J Biol Chem. 2009;284:22680-9.

15. Xiang M, Zeng Y, Yang R, Xu H, Chen Z, Zhong J, et al. U6 is not a suitable endogenous control for the quantification of circulating microRNAs. Biochem Biophys Res Commun. 2014;454:210-4.

16. Dong J, Lu M, He X, Xu J, Qin J, Cheng Z, et al. Identifying the role of microRNAs in spinal cord injury. Neurol Sci. 2014;35:1663-71.

17. Borgens RB, Liu-Snyder P. Understanding secondary injury. Q Rev Biol. 2012;87:89-127.

18. Yip PK, Malaspina A. Spinal cord trauma and the molecular point of no return. Mol Neurodegener. 2012;7:6.

19. Godwin JG, Ge X, Stephan K, Jurisch A, Tullius SG, Iacomini J. Identification of a microRNA signature of renal ischemia reperfusion injury. Proc Natl Acad Sci USA. 2010;107:14339-44.

20. Harrison EB, Hochfelder CG, Lamberty BG, Meays BM, Morsey BM, Kelso ML, et al. Traumatic brain injury increases levels of miR-21 in extracellular vesicles: implications for neuroinflammation. FEBS Open Bio. 2016;6:835-46.

21. Bhalala OG, Pan L, Sahni V, McGuire TL, Gruner K, Tourtellotte WG, et al. microRNA-21 regulates astrocytic response following spinal cord injury. J Neurosci. 2012;32:17935-47.

22. Duan X, Ji B, Wang X, Liu J, Zheng Z, Long C, et al. Expression of microRNA-1 and microRNA-21 in different protocols of ischemic conditioning in an isolated rat heart model. Cardiology. 2012;122:36-43.

23. Cheng Y, Liu X, Zhang S, Lin Y, Yang J, Zhang C. MicroRNA21 protects against the $\mathrm{H}(2) \mathrm{O}(2)$-induced injury on cardiac myocytes via its target gene PDCD4. J Mol Cell Cardiol. 2009;47:5-14.

24. Cheng Y, Zhu P, Yang J, Liu X, Dong S, Wang X, et al. Ischaemic preconditioning-regulated miR-21 protects heart against ischaemia/reperfusion injury via anti-apoptosis through its target PDCD4. Cardiovasc Res. 2010;87:431-9.

25. Lei P, Li Y, Chen X, Yang S, Zhang J. Microarray based analysis of microRNA expression in rat cerebral cortex after traumatic brain injury. Brain Res. 2009;1284:191-201.

26. Buller B, Liu X, Wang X, Zhang RL, Zhang L, Hozeska-Solgot A, et al. MicroRNA-21 protects neurons from ischemic death. FEBS J. 2010;277:4299-307.

27. Wang G, Wang JJ, Tang HM, To SS. Targeting strategies on miRNA-21 and PDCD4 for glioblastoma. Arch Biochem Biophys. 2015;580:64-74.

28. Ruan Q, Wang T, Kameswaran V, Wei Q, Johnson DS, Matschinsky $\mathrm{F}$, et al. The microRNA-21-PDCD4 axis prevents type 1 
diabetes by blocking pancreatic beta cell death. Proc Natl Acad Sci USA. 2011;108:12030-5.

29. Hannan JL, Matsui H, Sopko NA, Liu X, Weyne E, Albersen M, et al. Caspase-3 dependent nitrergic neuronal apoptosis following cavernous nerve injury is mediated via RhoA and ROCK activation in major pelvic ganglion. Sci Rep. 2016;6:29416.

30. Eto K, Goto S, Nakashima W, Ura Y, Abe SI. Loss of programmed cell death 4 induces apoptosis by promoting the translation of procaspase-3 mRNA. Cell Death Differ. 2012;19:573-81.
31. Liu G, Keeler BE, Zhukareva V, Houle JD. Cycling exercise affects the expression of apoptosis-associated microRNAs after spinal cord injury in rats. Exp Neurol. 2010;226:200-6.

32. van den Bogaart G, Meyenberg K, Risselada HJ, Amin H, Willig $\mathrm{KI}$, Hubrich BE, et al. Membrane protein sequestering by ionic protein-lipid interactions. Nature. 2011;479:552-5.

33. Tian R, Shi R. Dimercaprol is an acrolein scavenger that mitigates acrolein-mediated PC-12 cells toxicity and reduces acrolein in rat following spinal cord injury. $\mathrm{J}$ Neurochem. 2017;141:708-20. 